\title{
Dermatology
}

Dermatology 2012;224:22-23

DOI: $10.1159 / 000327079$

\section{Economies of Intraoperative Drawing: Blood, an Autologous Skin Marker}

\section{A.M. Skaria}

University of Berne, Berne, Switzerland

Key Words

Intraoperative marking $\cdot$ Medical costs

The background of this article is that in nearly all developed countries the evolution of medical costs is progressive. Any possible economies in the medical field are useful to slow down this evolution. In dermatological surgery the cost of material is important. This is mostly related on one side to the specificity of the material and on the other side - when used during the operation - to the fact that the material has to be specially sterilized and compatible for the use on humans.

Preoperative and intraoperative marking is an important point in skin surgery. Preoperative drawing can be effectuated in a nonsterile manner and with any skin marker which is not bearing the risk of tattooing. Several authors have underlined the risk of cross-infections of felt-tipped marker pens as well as the limited sterility under ink-marked epidermis [1-3]. On the other hand, intraoperative drawing needs special markers where the color does not bear the risk of tattooing; it should not be allergenic or irritating, and it should be sterile. Concerning sterility one might try to resterilize the used markers, which is not ideal; further, the marking pens withstand only 1 or 2 sterilizing cycles before drying out. Further sterilization of used skin marker pens is not recommended by the manufacturer and therefore bears a potential risk in any court case. Each marker costs about 3 USD and is worth saving.
Fig. 1. Blood is taken up by dipping a forceps into the blood.

Fig. 2. The forceps with the blood reservoir between its two branches.

Fig. 3. Drawing before harvesting a fullthickness skin graft.

Fig. 4. Drawing of a transposition flap.
1

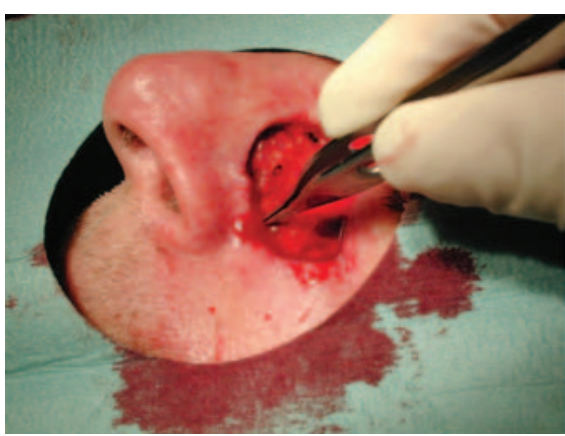

3

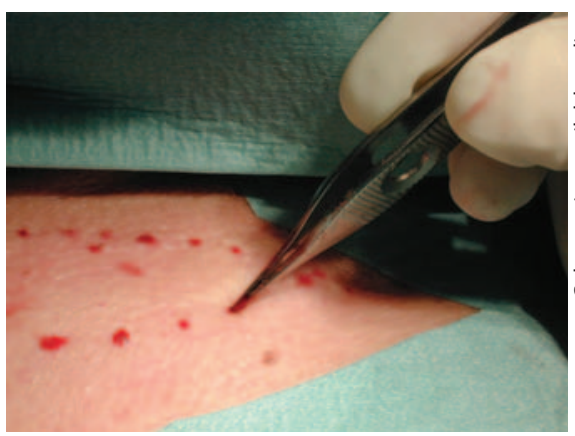

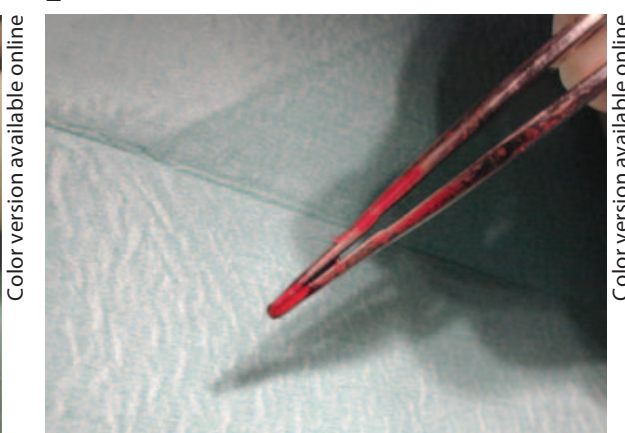

4

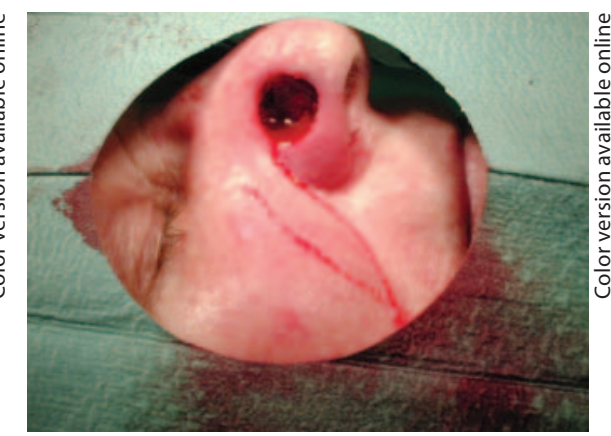


For my own use, I need intraoperative skin markers very rarely because the patient has a sufficient reservoir of marking substance: his own blood. When the tumor has been excised with Mohs surgery or with the necessary security margin, the created defect is bleeding. Even when electrocoagulation is performed, there is still some residual bleeding which is enough for skin marking. Therefore I use my forceps which acts as the feather of a fountain pen (fig. 1). Between the two branches of the forceps, the blood is captured and serves to mark the skin (fig. 2). For large drawings, or when there is only limited bleeding, small punctuations may be used (fig. 3); in small drawings one can draw a real line (fig. 4). After application one has to wait until the blood has dried before using any anesthetic which, when dripping on the wet blood, can wipe out your drawing. Strong bleeding should be stopped before marking as the bleeding might interfere with the drawing and wipe it out.

This kind of intraoperative marking may avoid the excessive use of marker pens and may help to diminish the costs of cutaneous surgery.
Disclosure Statement

No funding sources, no personal financial interest.

\section{References}

1 Sim DA, Feasey N, Wren S, Breathnach A, Thompson G: Cross infection risk of felt tipped marker pens in cataract surgery. Eye 2009;23: 1094-1097.

2 Tadiparthi S, Shokrollahi K, Cullan DB, Wongworawat MD: Sterility of the surgical site marking between the ink and the epidermis. J Am Coll Sorg 2007;205:319-321.

3 Juma A, Croall J: Using marker pens on patients: a potential source of cross infection with MRSA. Ann R Coll Surg Engl 2007;89:661-664.

Dr. A.M. Skaria

Mohs Surgeon, Centre de Dermatochirurgie

Rue de Lausanne 15

CH-1800 Vevey (Switzerland)

E-Mail skaria@vtxnet.ch 